\title{
On dissipative effects in solar prominences
}

\author{
I. Ballai ${ }^{\star}$ \\ Space \& Atmosphere Research Center, Dept. of Applied Mathematics, University of Sheffield, Hicks Building, \\ Hounsfield Road, Sheffield, S3 7RH, England, UK \\ Received 8 July 2003 / Accepted 11 September 2003

\begin{abstract}
The present paper aims to discuss the possible dissipative mechanisms acting in a coronal prominence. These mechanism are discussed within the context of spatial damping of linear compressional waves. The obtained results show that the dominant mechanism is thermal radiation provided the supposition of optically thin prominence is valid. Damping due to thermal conduction is a viable attenuation mechanism provided the wavelength of the waves is short.
\end{abstract}

Key words. Sun: atmosphere - prominences - evolution - magnetic fields - magnetohydrodynamics - numerical

\section{Introduction}

Prominences are cool and dense coronal objects seen in eclipse or coronograph images as bright features at the limb. On the disc they are seen in $H_{\alpha}$ pictures as thin, dark ribbons called filaments. Prominences are supported against gravity by the coronal magnetic field. They need this because their plasma is typically one hundred times denser than the coronal medium and because they extend in height over more than one hundred times the gravitational scale height of their cold plasma. The role of the coronal magnetic field is much broader: it controls not only the dynamics but also the thermodynamics (through thermal conduction and heating).

Recent observations show that all solar prominences oscillate, with characteristic periods from 1 minute to 1 hour with velocities up to $20 \mathrm{~km} \mathrm{~s}^{-1}$ (Balthasar et al. 1988, 1993; Engvold 2001). These oscillations are associated with the steady plasma flow present in these structures (for an extensive review on observational and theoretical aspects of waves in prominences, see Oliver \& Ballester 2002).

Molowny-Horas et al. (1999) using the VTT telescope at Sac Peak Observatory found velocity perturbations with periods between 28 and $95 \mathrm{~min}$ at different locations in a prominence. They observed that the amplitude of the oscillations decreases in time with damping times between 101 and $377 \mathrm{~min}$. The observations were fitted with a function proportional to $\cos (\omega t+\phi) \exp \left(-t / t_{\mathrm{D}}\right)$ where $\omega$ is the wave frequency, $\phi$ is the phase-shift and $t_{\mathrm{D}}$ is the damping time. Later, Terradas et al. (2001) using a simple model taking into account a radiative loss based on Newton's cooling law have shown that only slow waves are affected by damping, fast waves being almost undamped.

The existence of various dissipative mechanisms (e.g. viscosity, thermal and electrical conduction, radiative cooling,

\footnotetext{
* e-mail: i.ballai@sheffield.ac.uk
}

etc.) results in the dissipation of energy in magnetohydrodynamic (MHD) waves; the wave energy is consequently absorbed and the wave amplitude progressively attenuated. Dissipative mechanisms cause the amplitude of waves to vary slowly over a large scale. In our paper we are going to concentrate only on those oscillations which occur inside the prominence and we do not take into account the oscillations of the whole prominence structure (when the effect of the external coronal material has to be considered and waves propagate in a waveguide).

The present study aims to review some of the possible dissipative mechanisms which can work in the prominence to explain the spatial damping of compressional MHD waves. Special attention is paid to the isotropic and anisotropic cases.

\section{Dissipative mechanisms}

Before we embark on discussion of the possible dissipative mechanism acting in the solar prominences we have to introduce some basic values for the plasma and magnetic field. Throughout the paper we use the following values: magnetic field $B=5 \mathrm{G}$, average temperature $T=7000 \mathrm{~K}$, number density $n=2 \times 10^{10} \mathrm{~cm}^{-3}$. We suppose that the temperature of the electrons and ions are strongly correlated, so $\left|T_{\mathrm{e}}-T_{\mathrm{i}}\right| \ll T$.

First of all, the plasma parameter (Coulomb logarithm) $\ln \Lambda$ is given by

$\ln \Lambda=8 \ln 10+\frac{3}{2} \ln \left(\frac{T}{10^{6} \mathrm{~K}}\right)-\frac{1}{2} \ln \left(\frac{n}{10^{9} \mathrm{~cm}^{-3}}\right)$.

Using the given plasma parameters we obtain that $\ln \Lambda=9.49$. Another quantity used here is the electron thermal velocity defined by $v_{\text {th,e }} \approx 3 \times 10^{5} T^{1 / 2}=2.5 \times 10^{7} \mathrm{~cm} \mathrm{~s}^{-1}$. Since prominences are made from cool and dense material, the plasma can 
be considered as collisional. For the collisional assumption to be valid, the wave period must exceed the ion collisional time

$P>T^{3 / 2} n^{-1} \approx 5 \times 10^{-5} \mathrm{~s}$,

so plasma can be considered collisional for waves with frequencies smaller than $20 \mathrm{kHz}$. Since all the waves observed in prominences have frequencies much smaller than this value we can conclude that the prominence plasma can be considered as a collisional medium.

\subsection{Isotropic dissipation}

We suppose that all dissipative coefficients are constants and in this section the dissipation is isotropic. In what follows we concentrate on classical viscosity, electrical resistivity, thermal conduction and thermal (Newtonian) radiation.

Viscosity is a measure of friction, or resistance to flow, in a fluid. The viscosity of a plasma is due mainly to positive ions; viscous stresses due to electrons are generally negligible. The viscosity coefficient can be expressed as (Spitzer 1962)

$v_{\mathrm{v}}=1.32 \times 10^{11} \frac{T^{5 / 2}}{n \ln \Lambda} \mathrm{cm}^{2} / \mathrm{s}$

With the given parameters we obtain a coefficient of kinematic viscosity in the prominence of $2.8 \times 10^{9} \mathrm{~cm}^{2} \mathrm{~s}^{-1}$.

Electrical resistivity is defined as the ratio between the rate at which electrons in a unit volume gain momentum by impact with positive ions and the current density, i.e. it is related to the heating effect produced by the current flow in a resistive medium. Electrical conductivity can be expressed as

$\sigma=\frac{m_{\mathrm{e}} v_{\mathrm{th}, \mathrm{e}}^{3}}{e^{2} \ln \Lambda} \approx 6.5 \times 10^{12} \mathrm{~s}^{-1}$,

where $v_{\text {th,e }}$ has been defined before. Magnetic diffusivity, $v_{\mathrm{m}}$ is related to the electrical conductivity through $v_{\mathrm{m}}=c^{2} / 4 \pi \sigma$ where $c$ is the speed of light. For prominence values $v_{\mathrm{m}}=$ $1.1 \times 10^{7} \mathrm{~cm}^{2} \mathrm{~s}^{-1}$. In order to decide the importance of viscosity with respect to the magnetic diffusivity we can find that the ratio (magnetic Prandtl number) $v_{\mathrm{v}} / v_{\mathrm{m}}=2.5 \times 10^{2}$. We define the viscous (resistive) damping time as $t_{\mathrm{d}}=L^{2} / v_{\mathrm{v}}$ for viscosity and a similar relation for resistive decay but with different denominator and $L$ is a characteristic length. If we define the viscous damping length $\left(l_{\mathrm{d}}=t_{\mathrm{d}} c_{\mathrm{S}}\right.$ for slow waves and $l_{\mathrm{d}}=t_{\mathrm{d}} v_{\mathrm{A}}$ for Alfvén waves) as the distance that a wave can travel with the sound speed (for slow waves) or the Alfvén speed (for fast waves) in a viscous plasma, we obtain that it has to be larger than $1.5 \times 10^{15} \mathrm{~cm}$ for slow waves and $1.4 \times 10^{16} \mathrm{~cm}$ for fast waves. In the case of magnetic diffusivity we obtain a length of $4.3 \times 10^{17} \mathrm{~cm}$ for slow waves and $3.6 \times 10^{18} \mathrm{~cm}$ for fast waves. These lengths are much larger than the upper limit of the observed wavelengths $\left(2 \times 10^{9} \mathrm{~cm}\right)$ based on the results by Molowny-Horas et al. (1997). Therefore if kinematic viscosity or magnetic diffusivity are taken into account, slow and fast magnetoacoustic waves are damped over very large distances, i.e. these processes do not play an important role in the process of compressional wave damping in solar prominences.

Another very popular dissipative mechanism is radiative damping (due to its simple mathematical form in the
MHD equation) which owes its existence to the tendency of the plasma to smooth out any thermal perturbation. It takes place on the radiative relaxation time-scale, $\tau_{\mathrm{R}}$. When perturbations (oscillations) in the plasma occur over times longer than $\tau_{\mathrm{R}}$ the motion can not be considered adiabatic any longer and after a plasma element is displaced it comes into thermal equilibrium with its surroundings before the restoring forces have time to act.

Spiegel (1957) showed that if the motion occurs with a wavelength for which $\lambda \ll(\tilde{\kappa} \rho)^{-1}$, $(\tilde{\kappa}$ is the opacity or mass absorption coefficient and $\rho$ is the mass density), the plasma can be considered optically thin and the radiative damping time scale is given by

$\tau_{\mathrm{R}}=\frac{c_{\mathrm{v}}}{16 \sigma_{\mathrm{B}} \tilde{\kappa} T^{3}}$,

where $c_{\mathrm{V}}$ is the volumetric specific heat $\left(=13.8 \times 10^{7} \mathrm{erg}\right.$ $\mathrm{deg}^{-1} \mathrm{~mol}^{-1}$ ) and $\sigma_{\mathrm{B}}$ is the Stefan-Boltzmann constant.

The value of $\tau_{\mathrm{R}}$ is not known at all in prominences. This uncertainty arises due to the lack of the opacity measurements. Assuming optically thin radiation, Hood (1992) estimated a value for the radiative damping time of around $1 \mathrm{~s}$ which means that the plasma is optically thin for waves with wavelengths much smaller than $7 \times 10^{13} \mathrm{~cm}$. However, Terradas et al. (2001) pointed out that the radiative damping time can be quite different from 1 s. Weigert \& Wendker (1996) obtained that the average solar opacity per unit mass is $4 \times 10^{3} \mathrm{~cm}^{2} \mathrm{~g}^{-1}$. Using this value we obtain a radiative relaxation time of $1.1 \times 10^{-4} \mathrm{~s}$ and the plasma can be considered as optically thin for wavelengths much smaller than $8.3 \times 10^{9} \mathrm{~cm}$. This value is of the order of the lower limit of wavelengths obtained by Molowny-Horas et al. (1997), so for this opacity the plasma can be considered as optically thick and Eq. (2) does not apply.

Using these results we can conclude that for a radiative damping time of $1 \mathrm{~s}$ fast (slow) waves can have a significant damping for distances larger than $10^{7} \mathrm{~cm}\left(1.2 \times 10^{6} \mathrm{~cm}\right)$. If we take $\tau_{\mathrm{R}}=1.1 \times 10^{-4} \mathrm{~s}$, we obtain that these waves damp over distances larger than $10^{2} \mathrm{~cm}$. This lead to the conclusion that radiative damping is a viable damping mechanism for compressional MHD waves for radiative damping times of the order of a few seconds. However, we should remark that these estimations were not based on measured quantities.

\subsection{Anisotropic dissipation}

In this subsection we take into account dissipative effects for which the direction of the background magnetic field plays a key role. Two anisotropic dissipative effects are considered: viscosity and thermal conduction.

The condition of neglecting effects of anisotropy in viscosity for compressional waves is that the ion collisional rate $v_{\mathrm{i}}$ is much larger than the Larmor frequency $\omega_{\mathrm{Bi}}$ for ions (Lifshitz \& Pitaevsky 1981), i.e.

$\frac{B T^{3 / 2}}{n} \ll 5 \times 10^{-11}$,

where $T$ is measured in $\mathrm{eV}$. Introducing prominence parameters, we find that the inequality is fully satisfied, so the 
viscosity in prominences can be considered isotropic, mainly because of the very high density and very low temperature.

Thermal conduction reflects the tendency of the plasma to depart from the isentropic state. Thermal conductivity appears mainly due to electrons and in general, is a tensorial quantity. The heat flux vector can be written as

$\boldsymbol{q}=-\kappa_{\mathrm{T}} \nabla T$,

where $\boldsymbol{\kappa}_{\mathrm{T}}$ is the thermal conduction tensor. The energy loss function in the energy conservation equation is expressed as the divergence of the heat flux vector, which is proportional to

$\nabla_{\|} \cdot\left(\kappa_{\|} \nabla_{\|} T\right)+\nabla_{\perp} \cdot\left(\kappa_{\perp} \nabla_{\perp} T\right)$,

where the $\|$ and ${ }_{\perp}$ subscripts describe the values parallel and perpendicular to the magnetic field. The perpendicular conduction is due mainly to ions and in general for solar conditions it can be neglected (it can be shown that $\kappa_{\perp} / \kappa_{\|} \ll 1$ ) and $\kappa_{\|}=\kappa_{0} T^{5 / 2}$ with $\kappa_{0}=10^{-11}$.

Thermal conduction can be neglected in comparison with the adiabatic term in the energy equation if

$\frac{\rho^{\gamma}}{\gamma-1} \frac{D}{D t}\left(\frac{p}{\rho^{\gamma}}\right) \gg \nabla \cdot\left(\kappa_{0} T^{5 / 2} \nabla T\right)$,

where $D / D t=\partial / \partial t+v \cdot \nabla$ is the advective operator and $\nabla$ is the parallel operator. Assuming a typical length $(L)$ and timescale $(\tau)$, thermal conduction can be neglected if

$\frac{L^{2}}{\tau} \gg 5.6 \times 10^{8} \mathrm{~cm}^{2} \mathrm{~s}^{-1}$

Choosing a typical value for $\tau(\approx 40 \mathrm{~min}$, i.e. a typical wave period), we obtain that thermal conduction can be neglected for distances much larger than $1.1 \times 10^{6} \mathrm{~cm}$. Since the typical wavelengths are below $2 \times 10^{9} \mathrm{~cm}$, it is clear that only waves with very short wavelengths are affected by damping due to thermal conduction.

We should make one more remark here. Since in quiescent prominences $\omega_{\mathrm{e}} \tau_{\mathrm{e}} \gg 1\left(\approx 10^{3}, \omega_{\mathrm{e}}\right.$ is the electron gyrofrequency and $\tau_{\mathrm{e}}$ is the electron collisional time) the magnetic resistivity is anisotropic with $v_{\mathrm{m}}^{\perp}>v_{\mathrm{m}}^{\|}$. However, the consideration of anisotropy in magnetic resistivity does not introduce any significant change in our results since both coefficients are of the same order as the coefficient obtained in the isotropic limit.

\section{Conclusions}

The present letter has overviewed some of the possible dissipative effects acting in the prominences in conjunction with compressional MHD wave damping. We have considered isotropic (viscosity, magnetic diffusivity, radiative damping) and anisotropic (viscosity, thermal conduction) dissipative mechanisms. We have shown that some of these effects are not even applicable to prominences (anisotropic viscosity) and in general the considered effects can not explain the observed wave damping. Thermal radiation can explain the dampoing of linear compressional MHD waves but the uncertainty over the radiative damping time and the optical nature of the prominence makes it doubtful. Waves can damp due to the anisotropic thermal conduction if they have short wavelengths. This suggests some other damping mechanism could be considered such as wave leakage, ion-neutral damping, resonant absorption (the plasma is very likely to be strongly inhomogeneous due to very small scale height) may play an important role in the process of compressional MHD wave damping. The presented results will be exploited in a forthcoming paper where the damping of compressional waves is investigated taking into account the effects of the surrounding coronal plasma.

Acknowledgements. The author acknowledges the financial support by PPARC (UK) and NFS Hungary (OTKA, TO43741).

\section{References}

Balthasar, H., Stellmacher, G., \& Wiehr, E. 1988, A\&A, 204, 286

Balthasar, H., Wiehr, E., Schleicher, H., \& Wohl, H. 1993, A\&A, 227, 243

Braginskii, S. I. 1965, Rev. Plasma Phys., 1, 205

Engvold, O. 2001, in Workshop on MHD waves in astrophysical plasmas, ed. J. L. Ballester, \& B. Roberts, 123

Hood, A. W. 1992, Plasma Phys. Control. Fusion, 34, 411

Lifshitz, E. M. \& Pitaevsky, L. P. 1981, Physical Kinetics (Oxford: Pergamon Press)

Molowny-Horas, R., Wiehr, E., Balthasar, H., Oliver, R., \& Ballester, J. L. 1999, in JOSO Annual Report '98, ed. A. Antalová, H. Bathasar, H. \& T. Kučera (Astronomical Institute Tatrasnská Lomnica), 126

Molowny-Horas, R., Oliver, R., Ballester, J. L., \& Baudin, F. 1997, Sol. Phys., 172, 181

Oliver, R., \& Ballester, J. L. 2002, Sol. Phys., 206, 445

Spiegel, E. A. 1957, ApJ, 126, 202

Spitzer, L. 1962, Physics of Fully Ionized Gases (New York: Interscience)

Terradas, J., Oliver, R., \& Ballester, J. L. 2001, A\&A, 378, 635

Weigert, A., \& Wendker, H. J. 1996, Astronomie und Astrophysik. Ein Grundkurs (Weinheim: VCH Verlagsgessellshaft) 